\title{
THE ANALGESIC EFFICACY OF TAP BLOCK VERSUS EPIDURAL ANALGESIA: A SYSTEMATIC REVIEW AND META-ANALYSIS
}

\author{
Frank Zeiter, ${ }^{1}$ Moira Baeriswyl, ${ }^{1}$ Denis Piubellini, ${ }^{1}$ Kyle Robert Kirkham, ${ }^{2}$ Eric Albrecht ${ }^{1}$ \\ ${ }^{1}$ Department of Anaesthesia, Lausanne University Hospital, Lausanne, Switzerland. \\ 2Department of Anaesthesia, Toronto Western Hospital, University of Toronto, Toronto, Canada.
}

\section{Background and aim:}

TAP block has gained popularity in regional anaesthesia to provide postoperative analgesia but its advantage over epidural analgesia is disputed. The objective of this meta-analysis was to compare the analgesic efficacy of both techniques.

\section{Methods:}

We followed the PRISMA statement guidelines. Only trials comparing TAP block with epidural analgesia were included meta-analyses were performed following mostly a random-effects model. The primary outcome was pain score at rest (analogue scale, $0-10$ ) on postoperative day 1 analyzed in subgroups according to the population (children and adults). Secondary outcomes included rate of hypotension, length of stay, and functional outcomes (time to first bowel sound, time to first flatus). Ten controlled trials, including 505 patients, were identified.

\section{Results:}

Pain score at rest on postoperative day 1 was equivalent in TAP block and epidural analgesia groups in children (mean difference: $0.3 ; 95 \% \mathrm{Cl}:-0.1,0.6 ; \mathrm{I}^{2}=0 \% ; \mathrm{p}=0.15$ ) and in adults (mean difference: $0.5 ; 95 \% \mathrm{Cl}:-0.1,1.0$; $\mathrm{I}^{2}=81 \% ; \mathrm{p}=0.10$ ). The quality of evidence for our primary outcome was low according to the GRADE system, due to the risk of type II error. Rate of hypotension was higher in the epidural analgesia group (RR: $0.13 ; 95 \% \mathrm{Cl}: 0.04,0.38$; $1^{2}=0 \% ; p=0.0002$ ), while hospital length of stay was reduced in TAP block group (mean difference: -0.6 days; $95 \% \mathrm{Cl}:-0.9,-0.3$ days; $\mathrm{I}^{2}=0 \% ; \mathrm{p}<0.0001$ ), without impact on functional outcomes.

\section{Conclusions:}

There is low evidence that TAP block and epidural analgesia are equally effective in treating postoperative pain. Additional trials with robust methodology are required to better define the analgesic effect and the functional impact of each technique before recommending TAP block that is associated with less episodes of hypotension and reduced length of stay.

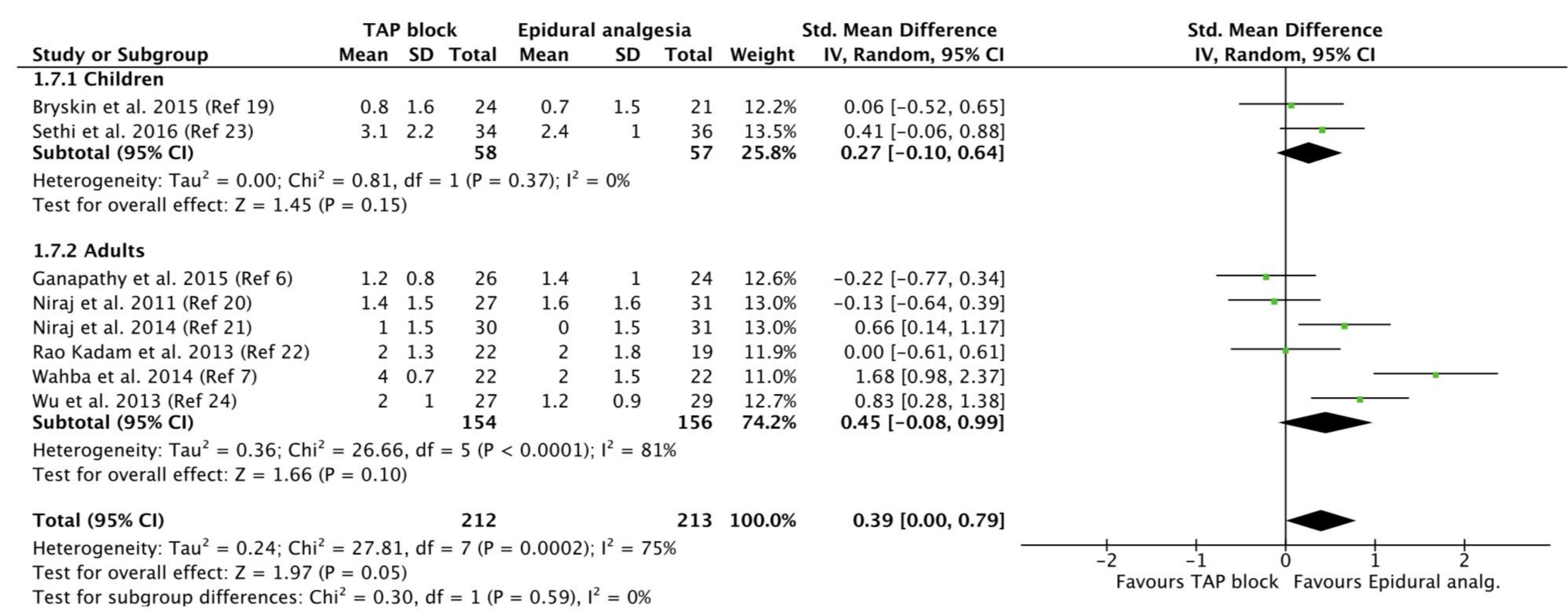

